\title{
Causa poco común de obstrucción nasosinusal recurrente: Enfermedad de Rosai-Dorfman
}

\section{Uncommon cause of recurrent sinus obstruction: Rosai-Dorfman disease}

\author{
Nicolás Albertz A¹, José Miguel Godoy $\mathbf{S}^{2}$, Luis Cabezas L², Luis Contreras $\mathbf{M}^{3}$.
}

\begin{abstract}
RESUMEN
La enfermedad de Rosai-Dorfman corresponde a una enfermedad rara con menos de 1.000 casos reportados. Sus características clínicas corresponden a un aumento de volumen en ganglios cervicales, sin embargo, presenta compromiso extranodal como única manifestación entre el $25 \%$ y $50 \%$ de los casos. La ubicación nasosinusal ha sido reportada previamente, pese a ello, es más frecuente encontrarla en otras ubicaciones. EI diagnóstico se basa en el estudio histopatológico encontrándose el fenómeno de emperipolesis, con células positivas a la proteína S-100. Esta enfermedad es frecuentemente confundida con neoplasias malignas, por lo que debe ser reconocida y sospechada.

Se presenta el caso de un paciente de 15 años con historia obstrucción nasal de larga data, con múltiples recurrencias de la enfermedad de Rosai-Dorfman, demostrada mediante histopatología.
\end{abstract}

Palabras clave: Rosai-Dorfman, emperipolesis, obstrucción nasal, recurrencias.

\begin{abstract}
The Rosai-Dorfman disease corresponds to a rare disease with less than 1,000 reported cases. Their clinical characteristics correspond to a volume increase in cervical nodes, There is an extranodal involvement as the only manifestation of between $25 \%$ and $50 \%$ of cases. The sinonasal location has been previously reported. Diagnosis is based on histopathology with the phenomenon of emperipolesis with cells positive for $S-100$ protein. This disease is often confused with malignancy, so it must be recognized and suspected. A case of a patient 15 years with a history of longstanding nasal obstruction, with multiple recurrences of Rosai-Dorfman disease, demonstrated by histopathology.
\end{abstract}

${ }^{1}$ Médico, Programa Magister en Salud Pública Universidad de Chile, Becario CONICYT. Santiago, Chile.

${ }^{2}$ Médico, Departamento de Otorrinolaringología, Clínica Las Condes. Santiago, Chile.

${ }_{3}^{3}$ Médico, Laboratorio de Anatomía Patológica, Clínica Las Condes. Santiago, Chile. 


\section{INTRODUCCIÓN}

La histiocitosis sinusoidal con linfoadenopatías masivas, condición conocida como enfermedad de Rosai y Dorfman, fue reconocida como entidad patológica independiente en 1969 por ambos autores, sin embargo, previamente, en 1947 Robb Smith ya la había descrito en niños ${ }^{1}$. Esta corresponde a una enfermedad rara con menos de 1.000 casos reportados en la literatura mundial, pudiendo presentarse durante toda la vida, con predilección por la primera y segunda década ${ }^{2,3}$. Sus características clínicas corresponden al aumento de volumen en ganglios cervicales no doloroso, fiebre y velocidad de sedimentación aumentada. Su compromiso extranodal ocurre entre $25 \%$ y $50 \%$ de los casos, presentándose como única manifestación de la enfermedad en casos raros ${ }^{4-6}$.

La ubicación nasosinusal ha sido reportada previamente ${ }^{5}$, sin embargo, como manifestación única de enfermedad es más frecuente encontrarla en el sistema nervioso central, alteraciones cutáneas, óseas, en sistema genitourinario, tracto respiratorio bajo y tejidos blandos ${ }^{7,8}$.

El diagnóstico de la enfermedad se basa en el estudio histológico del tejido comprometido. En éste se puede encontrar una proliferación benigna de tejido fibroso y hematopoyético, acompañado de un infiltrado mixto de células plasmáticas y abundantes células histiocíticas que demuestran fagocitosis de linfocitos, o emperipolesis. Además es característica la presencia de histiocitos inmunorreactivos a la proteína S-100, que se utiliza para confirmar el diagnóstico $0^{7,9-11}$.

No existe consenso respecto a su etiología, siendo ésta aún incierta, sin embargo, se han postulado deficiencias inmunológicas, alteraciones genéticas o bien etiologías infecciosas².

A propósito de esta enfermedad poco común y a la experiencia clínica adquirida, se presenta la siguiente situación clínica.

\section{CASO CLÍNICO}

Paciente de 15 años, sexo masculino, consulta por una historia de obstrucción nasal intermitente de años de evolución, sin resolución pese a múltiples consultas y tratamientos. En agosto de 2007 consulta en pediatría general por síntomas similares a una rinofaringitis aguda, siendo tratado en ese entonces con medidas generales, corticoides inhalatorios y antihistamínicos por el período agudo. Dada la persistencia de su sintomatología se realizó un estudio para rinitis alérgica con un prick test y estudio de rinosinusitis con tomografía computarizada de cavidades paranasales, sin resultados positivos en ninguno de dichos estudios.

Luego de una evolución tórpida de su obstrucción nasal, en febrero de 2008 consulta en otorrinolaringología por persistencia de la sintomatología de manera intermitente. Se practicó nuevamente una tomografía computarizada y además una resonancia magnética de cavidades paranasales (Figuras 1 y 2 ), evidenciando una masa rinosinusal a nivel del tabique nasal. Dada la sintomatología del paciente, asociada a las imágenes encontradas en ese entonces, se decide intervenir quirúrgicamente en mayo de 2008, planteando el diagnóstico de probable neoplasia de fosa nasal. Se procede a resecar la masa a nivel de tabique nasal en $90 \%$ de su extensión en una primera instancia.

Pese a estos antecedentes, la biopsia de la primera intervención quirúrgica resulta negativa para neoplasias. El paciente evoluciona con remisión parcial de su sintomatología, por lo que aproximadamente al año de la primera intervención se reestudia el caso por recurrencia de su obstrucción nasal, evidenciando un nuevo crecimiento tumoral a nivel de tabique nasal (Figura 3). Por este motivo en agosto de 2009 se debe reintervenir quirúrgicamente con resección parcial de la masa de tabique nasal, dando el diagnóstico histológico inicial de inflamación inespecífica. Dada la inconformidad de dicho diagnóstico se decidió enviar la biopsia a revisión y posteriormente a un centro de anatomía patológica internacional, la cual sugiere una posible enfermedad de Rosai-Dorfman.

Posteriormente a la segunda intervención quirúrgica evoluciona asintomático por 6 a 8 meses, para luego reaparecer la obstrucción nasal con mayor intensidad, oportunidad en que se atiende en el Departamento de Otorrinolaringología de Clínica Las Condes. En la evaluación clínica inicial, en la rinoscopía anterior se evidencia una obstrucción nasal completa por ambas fosas nasales que impiden una mayor visualización (Figuras 4 y 5), por lo que se plantea una recidiva de su enferme- 

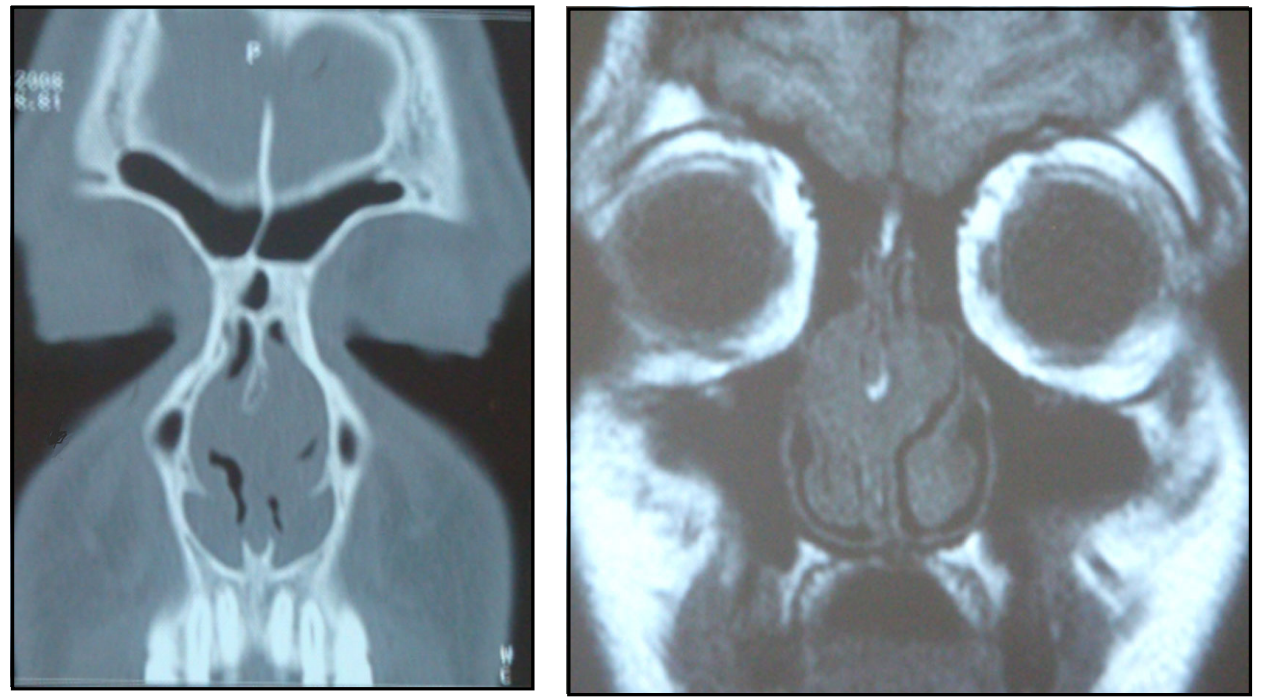

Figuras 1 y 2. Cortes coronales de TC y RM de cavidades paranasales que evidencian masa tumoral a nivel de tabique nasal.

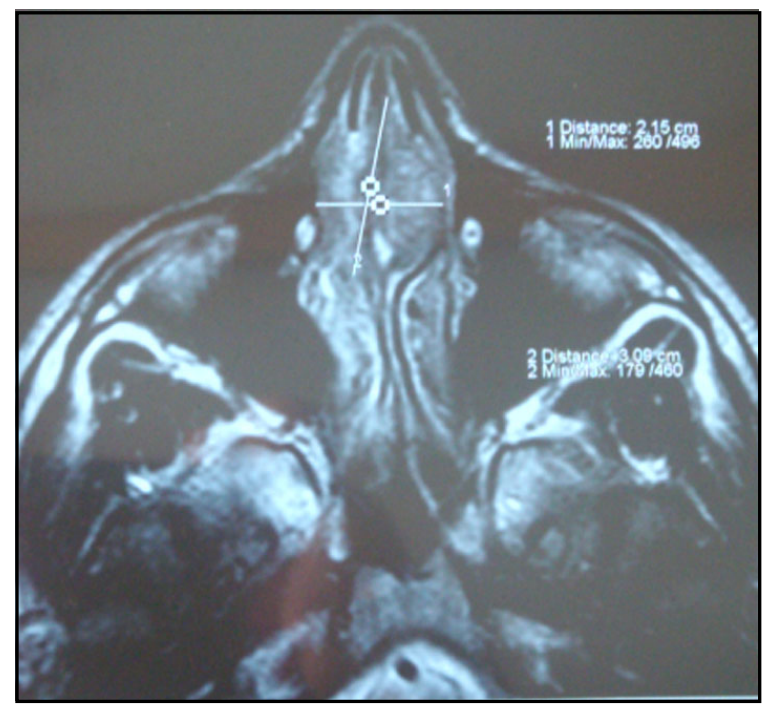

Figura 3. Corte transversal de TC de cavidades paranasales, con masa tumoral en tabique nasal.

dad de base. Luego de realizar un estudio preoperatorio completo, se decide practicar una resección amplia de tabique nasal y cornetes, oportunidad en que se eliminó por completo la masa obstructiva.

A pesar que en esa última intervención hubo una resección macroscópica completa, un año después reaparece la sintomatología con la obs- trucción nasal intermitente. Nuevamente se reestudia el caso y se decide reintervenir quirúrgicamente. En los estudios histopatológicos concomitantes, persiste el diagnóstico de enfermedad de Rosai-Dorfman con la característica emperipolesis (Figura 6) asociado a una proteína S-100 positiva (Figura 7), siendo a su vez descartados los posibles diagnósticos diferenciales. A la 

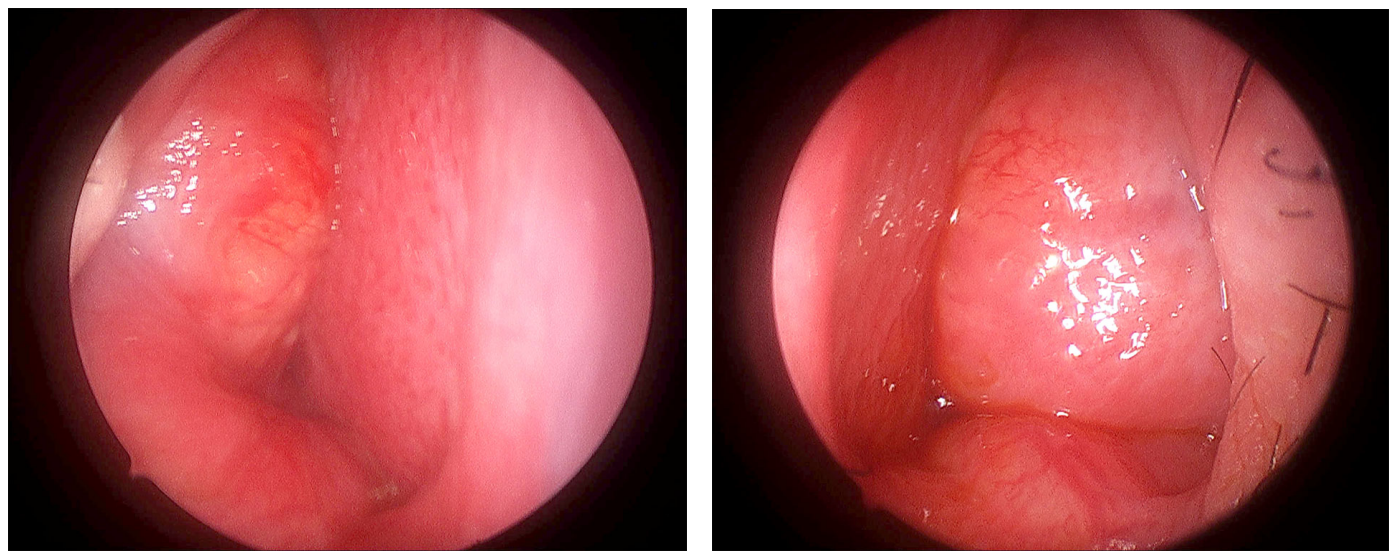

Figuras 4 y 5. Rinoscopía anterior que evidencia obstrucción nasal en ambas fosas nasales.

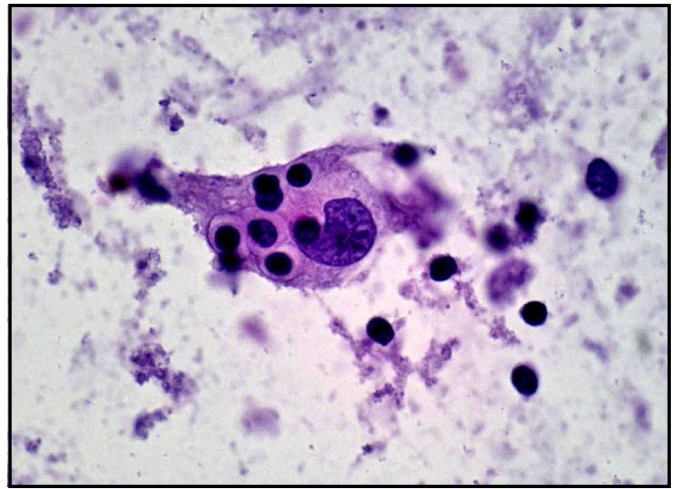

Figura 6. Impronta citológica obtenida durante biopsia intraoperatoria. Macrófago con fagocitosis de múltiples linfocitos (HE 400X).

fecha a nuestro paciente se le han realizado 4 cirugías de resección nasosinusales, encontrándose asintomático desde su última intervención hace 10 meses, con un tratamiento de mantención consistente en inhalador nasal de limpieza, antihistamínicos y corticoides nasales.

\section{DISCUSIÓN}

La enfermedad de Rosai-Dorfman es una entidad poco común que pertenece a las enfermedades

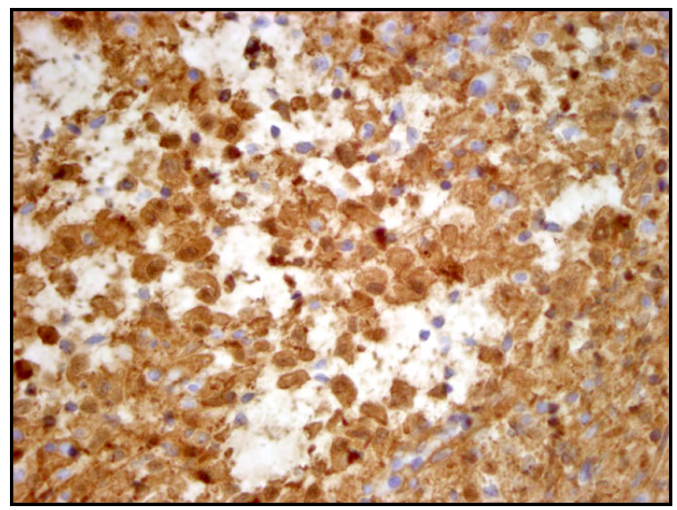

Figura 7. Macrófagos se tiñen intensamente positivos en forma difusa con proteína S100 (200X).

histiocíticas no malignas, con un aumento patológico de histiocitos, principalmente fagocitos mononucleares y células presentadoras de antígenos. Su etiología permanece desconocida habiéndose postulado teorías genéticas, infecciosas, inflamatorias y autoinmunes ${ }^{12,13}$.

Así como existe una falta de consenso en su etiología, tampoco disponemos de un acuerdo para obtener un tratamiento efectivo y estandarizado en todos los casos. Se han intentado diversas estrategias de acuerdo a la sintomatología específica que presente cada paciente, sin embargo, éstas sólo 
son capaces de disminuir el síntoma en particular y no erradicar la enfermedad ${ }^{14}$. Dada la escasa información certera del manejo de esta patología, existen grupos que plantean la observación clínica en los casos menos sintomáticos, hasta otros que plantean combinaciones de cirugía, quimio y radioterapia, en los casos más agresivos ${ }^{15,16}$.

Por otro lado es fundamental mencionar que esta enfermedad es frecuentemente confundida con neoplasias malignas, sin embargo, su evolución clínica es variable. Esta puede ir desde la regresión espontánea, linfadenopatías persistentes o bien llegar hasta recurrencias sintomatológicas intermitentes según su ubicación. También cabe destacar la posibilidad que la enfermedad se presente sin la presencia de las linfadenopatías, tal como ocurre en nuestro caso clínic ${ }^{17}$. Por este motivo, si bien se trata de una enfermedad rara, ésta debe ser considerada y se debe tener un alto índice de sospecha frente a casos de obstrucción nasal persistentes sin etiología precisa.

En el caso particular de nuestro paciente se han evaluado diferentes opciones de manejo por equipos tratantes multidisciplinarios, sin embargo, hasta la fecha ninguno ha logrado impedir las recurrencias. Han pasado 10 meses desde la última intervención quirúrgica con buenos resultados y estamos esperanzados en el éxito logrado hasta el momento, sin embargo, debemos tener cautela y realizar controles clínicos seriados en el tiempo que nos permitan esclarecer el mejor manejo de nuestro paciente.

\section{BIBLIOGRAFÍA}

1. Rosal J, Dorfman RF. Sinus histiocytosis with massive lymphadenopathy. A newly recognized benign clinicopathological entity. Arch Pathol 1969; 87(1): 63-70.

2. Henter JI, Tondini C, Pritchard J. Histiocyte disorders. Crit Rev Oncol Hematol 2004; 50(2): 157-74.

3. Moore JC, Zhao X, Nelson El. Concomitant sinus histiocytosis with massive lymphadenopathy (Rosai-Dorfman Disease) and diffuse large Bcell lymphoma: a case report. J Med Case Reports 2008; 2: 70.

4. Ferrer Rodríguez A, Agut Fuster MA, Jiménez A, Ramos Martínez MJ, del Campo Biosca J, Agulles
Fornes MJ, et al. Enfermedad de RosaiDorfman (histiocitosis sinusal con linfadenopatías masivas): a propósito de un caso. Acta Otorrinolaringol Esp 2003; 54(5): 384-7.

5. Córdova G, Paz A, Benitez S, Caballero J. Enfermedad de Rosai-Dorfman. Rev Otorrinolaringol Cir Cabeza Cuello 2004; 64: 39-42.

6. Wenig BM, Abbondanzo SL, Childers El, Kapadia SB, HeFFNER DR. Extranodal sinus histiocytosis with massive lymphadenopathy (RosaiDorfman disease) of the head and neck. Hum Pathol 1993; 24(5): 483-92.

7. Foucar E, Rosal J, Dorfman R. Sinus histiocytosis with massive lymphadenopathy (Rosai-Dorfman disease): review of the entity. Semin Diagn Pathol 1990; 7(1): 19-73.

8. Mir R, Aftalion B, Kahn LB. Sinus histiocytosis with massive lymphadenopathy and unusual extranodal manifestations. Arch Pathol Lab Med 1985; 109(9): 867-70.

9. Paulli M, Rosso R, Kindl S, Boveri E, Marocolo D, CHIODA C, ET AL. Immunophenotypic characterization of the cell infiltrate in five cases of sinus histiocytosis with massive lymphadenopathy (Rosai-Dorfman disease). Hum Pathol 1992; 23(6): 647-54.

10. Pulsoni A, Anghel G, Falcucci P, Matera R, Pescarmona E, Ribersani M, et al. Treatment of sinus histiocytosis with massive lymphadenopathy (Rosai-Dorfman disease): report of a case and literature review. $A m \mathrm{~J}$ Hematol 2002; 69(1): 67-71.

11. Bonetti F, Chilosi M, Menestrina F, Scarpa $A$, Pelicc PG, Amorosi E, et AL. Immunohistological analysis of Rosai-Dorfman histiocytosis. A disease of S-100 + CD1-histiocytes. Virchows Arch A Pathol Anat Histopathol 1987; 411(2): 129-35.

12. Morgan NV, Morris MR, Cangul H, Gleeson D, Straatman-Iwanowska A, Davies $\mathrm{N}$, et al. Mutations in SLC29A3, encoding an equilibrative nucleoside transporter ENT3, cause a familial histiocytosis syndrome (Faisalabad histiocytosis) and familial RosaiDorfman disease. PLoS Genet 2010; 6(2): e1000833.

13. Mehraein $Y$, Wagner M, Remberger K, Fuzesi L, Middel P, Kaptur S, et al. Parvovirus B19 
detected in Rosai-Dorfman disease in nodal and extranodal manifestations. J Clin Pathol 2006; 59(12): 1320-6.

14. Picco P, Buoncompagni A, Pistola V, Di Rocco M, Giustard A, Brisigotti M, et al. Diagnostic difficulties and positive therapeutic response in a patient with sinus histiocytosis with massive lymphadenopathy. Eur J Pediatr 1993; 152(8): 699.

15. Aouba A, Terrier B, Vasiliu V, Candon S, Brousse $\mathrm{N}$, Varet B, et al. Dramatic clinical efficacy of cladribine in Rosai-Dorfman disease and evolution of the cytokine profile: towards a new therapeutic approach. Haematologica 2006; 91(12 Suppl): ECR52.

16. Raveenthiran V, Dhanalakshmi M, Hayavadana Rao PV, Viswanathan P. Rosai-Dorfman disease: report of a 3-year-old girl with critical review of treatment options. Eur J Pediatr Surg 2003; 13(5): 350-4.

17. Imabuchi $\mathrm{H}$, Kakihara T, Tanaka A, Uchiyama M, ShIBUYA H, UMmeZU H. Congenital Rosai-Dorfman disease without lymphadenopathy. Pediatr Pathol Mol Med 2003; 22(5): 399-403. 